\title{
Impact of postdilatation on performance of bioresorbable vascular scaffolds in patients with acute coronary syndrome compared with everolimus-eluting stents: A propensity score-matched analysis from a multicenter "real-world" registry
}

Yoichi Imori ${ }^{1 *}$, Fabrizio D'Ascenzo ${ }^{1,2 *}$, Tommaso Gori ${ }^{3}$, Thomas Münzel ${ }^{3}$, Fabrizio Ugo ${ }^{4}$, Gianluca Campo ${ }^{5,6}$, Enrico Cerrato ${ }^{7}$, L. Christian Napp ${ }^{8}$, Mario Iannaccone, Jelena R. Ghadri ${ }^{1}$, Elycia Kazemian ${ }^{1}$, Ronald K. Binder ${ }^{1}$, Milosz Jaguszewski ${ }^{1}$, Adam Csordas $^{1}$, Piera Capasso ${ }^{4}$, Simone Biscaglia, ${ }^{5,6}$, Federico Conrotto ${ }^{2}$, Ferdinando Varbella ${ }^{7}$, Roberto Garbo ${ }^{4}$, Fiorenzo Gaita ${ }^{2}$, Paul Erne ${ }^{9,10}$, Thomas F. Lüscher ${ }^{1}$, Claudio Moretti ${ }^{2}$, Antonio H. Frangieh ${ }^{1 *}$, Christian Templin ${ }^{1 *}$

${ }^{1}$ University Heart Center, Department of Cardiology, University Hospital Zurich, Switzerland ${ }^{2}$ Dipartimento di Scienze Mediche, Divisione di Cardiologia, Città della Salute e della Scienza, Turin, Italy ${ }^{3}$ Medizinische Klinik und Poliklinik-Kardiologie, Angiologie und Internistische Intensivmedizin, University Medical Center, Mainz, Germany

${ }^{4}$ Interventional Cardiology Department, San Giovanni Bosco Hospital, Turin, Italy ${ }^{5}$ Cardiovascular Institute, Azienda Ospedaliero-Universitaria S. Anna, Cona, FE, Italy ${ }^{6}$ Laboratorio per le Tecnologie delle Terapie Avanzate (LTTA) Center, Ferrara, Italy

${ }^{7}$ Cardiology Department, Ospedale degli Infermi, Rivoli TO, Italy

${ }^{8}$ Department of Cardiology and Angiology, Hannover Medical School, Hannover, Germany

${ }^{9}$ Heart Centre Lucerne, Luzerner Kantonsspital, Lucerne, Switzerland

${ }^{10}$ Department of Cardiology, Klinik St. Anna, Lucerne, Switzerland

\begin{abstract}
Background: Safety and efficacy of bioresorbable vascular scaffolds (BRS) and the role of postdilatation on outcome in acute coronary syndrome (ACS) patients compared with those of everolimus-eluting stents (EES) remain unknown. The aim of the study is to compare the safety and efficacy of BRS with EES in ACS and to investigate the role of BRS postdilatation. Methods: Consecutive ACS patients undergoing BRS implantation in 8 centers were compared with those with EES before and after propensity score matching. Major adverse cardiac event (MACE), myocardial infarction, and target lesion revascularization (TLR) were the primary endpoint. Sensitivity analysis was performed according to postdilatation after BRS
\end{abstract}

Address for correspondence: Christian Templin, MD, PhD, University Heart Center, Department of Cardiology,

University Hospital of Zurich, Raemistr. 100, 8091 Zürich, Switzerland, tel: +41 (0)44 255 9585, fax: +41 (0)44 255 4401, e-mail: christian.templin@usz.ch; Fabrizio D’Ascenzo, MD, Division of Cardiology, Department of Medical Science, University of Turin, Città Della Salute e Della Scienza, Turin, Italy, e-mail: fabrizio.dascenzo@gmail.com *Contributed equally to this work 
implantation. We enrolled 303 BRS and 748 EES patients; 215 from each group were compared after matching, and 117 (55.2\%) BRS patients were treated with postdilatation.

Results: After a median follow-up of 24.0 months, MACE rates were higher in BRS patients than in EES patients (9.3\% vs. 4.7\%, $p<0.001)$, mainly driven by TLR (6.1\% vs. $1.9 \%$, $p<0.001)$. Stent thrombosis increased in the BRS group (2.8\% vs. $0.9 \%, p=0.01)$. However, after sensitivity analysis, MACE rates in BRS patients with postdilatation were signifcantly lower than in those without, comparable to EES patients $(6.0 \%$ vs. $12.6 \%$ vs. $4.7 \%$, $p<0.001)$. The same trend was observed for TLR (3.4\% vs. $8.4 \%$ vs. $1.9 \%, p<0.001)$. Stent thrombosis rates were higher in both the BRS groups than in EES patients (2.6\% vs. $3.2 \%$ vs. $0.9 \%, p=0.045)$.

Conclusions: Postdilatation appears effective when using BRS in ACS patients. MACE rates are comparable to those of EES, although scaffold thrombosis is not negligible. Randomized prospective studies are required for further investigation. (Cardiol J 2016; 23, 4: 374-383)

Key words: angina pectoris, myocardial infarction, coronary restenosis, thrombosis, cardiac death

\section{Introduction}

Patients with acute coronary syndrome (ACS) represent a peculiar case for interventional cardiology because of the high risk of thrombosis and challenging lesions in unstable presentation $[1,2]$. Second generation drug-eluting stents (DES) such as everolimus-eluting stents (EES) and zotarolimus-eluting stents are the treatment of choice for ACS patients because of their superiority in terms of reducing subsequent revascularizations [3]. The most critical limit of DES as metal stent is the permanent caging of the coronary vessel, which increases the risk of neointima proliferation and very late stent thrombosis (ST) and may render surgical revascularization no longer feasible for patients with severe diffuse disease [4-7]. Recently, bioresorbable vascular scaffolds (BRS) were introduced, which provide temporary vessel scaffolding with drug delivery capacity but do not carry the limitations of a permanent metallic stent in the long term $[8,9]$. Prospective studies demonstrated BRS safety and efficacy on selected patients [10-12], but some studies have reported increased ST rates [13-19]. However, data on a large sample of ACS patients are still lacking [15, $17,20-24]$, and only few studies have evaluated the impact of lesion preparation and postdilatation of BRS [25-27]. The aim of the present study was to compare the safety and efficacy of BRS with those of EES in patients with ACS and to investigate the potential role of BRS postdilatation on outcome.

\section{Methods}

All consecutive BRS patients in 8 high-volume centers from Switzerland, Italy, and Germany (Supplementary Methods: see journal website) were enrolled and compared with consecutive patients from the Zurich ACS registry treated with EES at the Universität Spital Zurich, Switzerland. Patients admitted with a diagnosis of ACS according to European Society of Cardiology guidelines (unstable angina, non-ST segment elevation myocardial infarction, and ST segment elevation myocardial infarction [STEMI]) [28]. Baseline characteristics (age, gender, cardiovascular risk factors, and clinical presentation) and procedural features (target vessel; site and type of lesion [29]; and number, length, and diameter of stents) were collected. For BRS patients, the number and atmospheres of pre- and postdilatation and use of intravascular ultrasound (IVUS) or optical coherence tomography (OCT) were also recorded. Ethics approval was obtained from the Institutional Review Committees in the all institutions. All studies been performed in accordance with the ethical standards laid down in the 1964 Declaration of Helsinki and its later amendments. As this was retrospective study, no informed consent was required.

All percutaneous coronary interventions (PCI) were performed according to current standards, with mandatory predilatation and scaffold implantation at pressure below the indicated burst pressure. Specific treatment strategies including postdilatation were performed at physician's dis- 
cretion. Unless patients were already on chronic aspirin therapy, a loading dose of $250-500 \mathrm{mg}$ aspirin was administered before PCI, followed by $75-100 \mathrm{mg}$ once daily (od) dose indefinitely thereafter. Similarly, unless patients were already on chronic maintenance therapy, a loading dose of clopidogrel $(600 \mathrm{mg})$, prasugrel $(60 \mathrm{mg})$, or ticagrelor $(180 \mathrm{mg})$ was administered before or immediately after PCI, followed by a maintenance dose of clopidogrel (75 $\mathrm{mg}$ od), prasugrel (10 mg od), or ticagrelor (90 mg twice daily) for 12 months. The use of glycoprotein $\mathrm{IIb} / \mathrm{III}$ inhibitors was at the physician's discretion.

Patients either received treatment with BRS (Absorb, Abbott Vascular, Santa Clara, CA, USA) or treatment with EES (Xience, Abbott Vascular, Santa Clara, CA, USA). Postdilatation data recorded included the kind of balloon, size (mm), atmospheres of postdilatation, and final size reached. Postdilatation was deemed effective if a noncompliant balloon was inflated to no more than $0.5 \mathrm{~mm}$ larger than BRS.

Major adverse cardiac event (MACE), a composite of death from any cause, myocardial infarction (MI), and clinically driven target lesion revascularization (TLR), was the primary end point, whereas all components and clinically driven target vessel revascularization (TVR) as well as ST were secondary end points. Specifically, Academic Research Consortium [30] differentiated ST as early (0-30 days), late (31-365 days), or very late (> 365 days). Definite ST was defined as angiographically or pathologically proven ST, whereas probable ST was defined as any unexplained death within the first 30 days after stenting or any MI, irrespective of time after procedure, related to documented acute ischemia in the territory of the implanted stent, without angiographic confirmation of ST, and in the absence of any other obvious cause.

\section{Statistical analysis}

Continuous variables are presented as mean \pm standard deviation or median with interquartile range, and categorical variables are presented as frequency (\%). Categorical variables were compared using Fisher's exact test. Parametric distribution of continuous variables was tested graphically and with Hosmer-Lemeshow test, and appropriate analyses were used in accordance with the results. For propensity score, first logistic regression analysis was performed for all baseline features that differed between BRS and EES groups and for age, gender, diabetes mellitus, presence of type $\mathrm{C}$ lesions, and admission diagnosis. Match- ing was computed after division into quintiles and methods of nearest neighbor on the estimated propensity score [26]. Calibration was tested using Hosmer-Lemeshow test, and accuracy was assessed with area under the curve. Standardized differences were evaluated before and after matching to evaluate the performance of the model. The cumulative incidences of the primary endpoint and the secondary endpoints were calculated using the Kaplan-Meier method with median follow-up analysis, and differences among the groups were analyzed using a stratified log-rank test. All statistical analyses were performed using SPSS 21, and differences were considered significant at $\alpha=0.05$. Plots were created using Graphpad Prism 6.

\section{Results}

In total, $303 \mathrm{BRS}$ and 748 EES patients were enrolled (Fig. 1).

Clinical presentation significantly differed between the groups (STEMI: $39.6 \%$ vs. $54.8 \%$, $\mathrm{p}<0.001$ ) (Table 1).

At baseline, BRS patients were more likely to suffer from hypertension $(60.3 \%$ vs. $52.6 \%$, $\mathrm{p}=0.03)$. Coronary angiography showed that left anterior descending coronary artery was the most frequently treated vessel (54.6\% vs. $50.4 \%$ ), followed by the right coronary artery $(27.0 \%$ vs. $27.2 \%, \mathrm{p}=0.32$ ). Type $\mathrm{C}$ lesions were present in $41.9 \%$ and $38.3 \%(p=0.40)$ of the patients with a mean stent diameter of $3.1 \pm 0.4 \mathrm{~mm}$ vs. $3.0 \pm$ $\pm 0.4 \mathrm{~mm}(\mathrm{p}<0.001)$ and a mean length of $20.6 \pm$ $\pm 5.2 \mathrm{~mm}$ vs. $20.0 \pm 5.4 \mathrm{~mm}(\mathrm{p}=0.07)$, respectively (Table 1). Postdilatation was performed in $49.2 \%$ of BRS patients $(\mathrm{n}=148)$ with noncompliant balloons and a mean pressure of $20 \pm 6 \mathrm{~atm}$. Intracoronary imaging was performed on $20.0 \%$ (IVUS) and $3.0 \%$ (OCT) of BRS patients. After a median follow-up of 24.0 (14.3-24.0) months, Kaplan-Meier curves showed that the rates of MACE were significantly higher in BRS patients ( $8.9 \%$ vs. $5.9 \%, \mathrm{p}<0.001)$, primarily due to an increased risk of TLR ( $5.3 \%$ vs. $1.6 \%, \mathrm{p}<0.001)$ and $\mathrm{MI}(4.3 \%$ vs. $3.1 \%, \mathrm{p}=0.02)$. Death rate was not different between the groups (2.9\% vs. $1.7 \%, \mathrm{p}=0.45)$. ST was higher in the BRS group (2.3\% vs. $1.2 \%, \mathrm{p}=0.03$ ) (Fig. 2) (Suppl. Table 1 and Suppl. Fig. 1A: see journal website).

After propensity score matching, 215 BRS and EES patients, each, with similar clinical presentation, baseline risk factors, and angiographic features were selected (Fig. 1, Table 2).

Thus, the results of the initial study on the overall population were confirmed. In fact, 


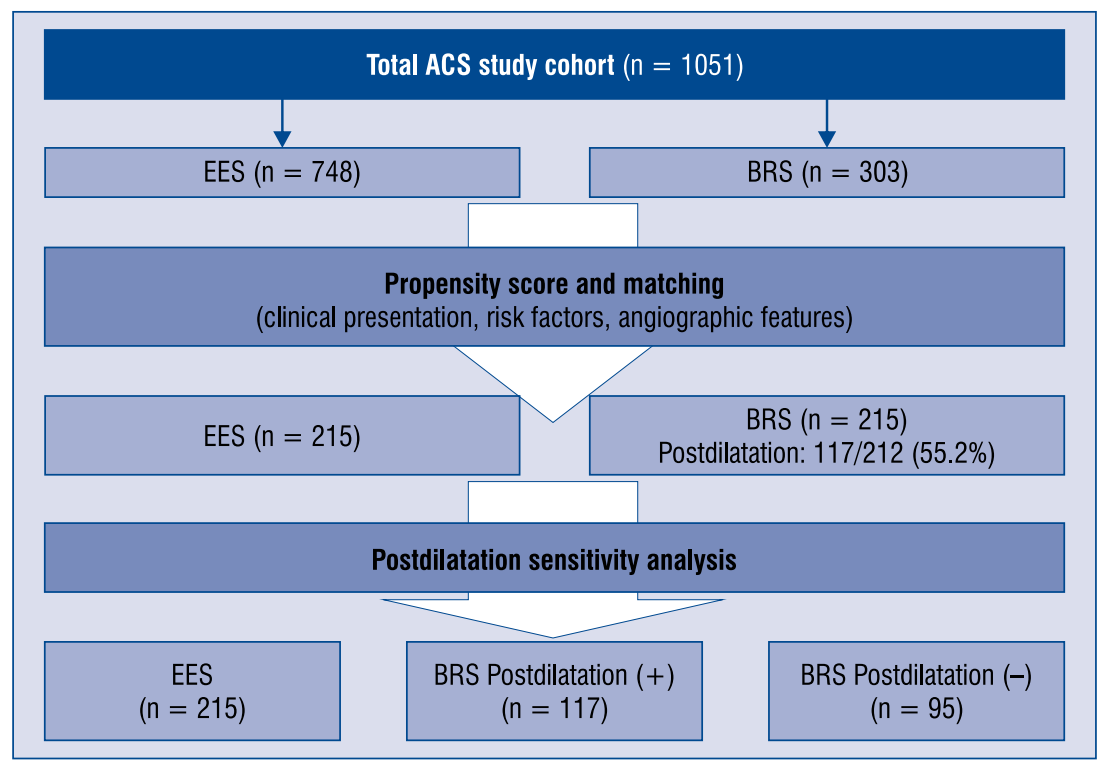

Figure 1. Flow diagram of the study; ACS — acute coronary syndrome; BRS — bioresorbable vascular scaffold; EES - everolimus-eluting stent.

Table 1. Baseline characteristics of the total study cohort (prematching).

\begin{tabular}{|c|c|c|c|}
\hline & BRS & EES & $\mathbf{P}$ \\
\hline Females & $68 / 303(22.4 \%)$ & $140 / 748(18.7 \%)$ & 0.17 \\
\hline Age [years] & $60.7 \pm 12.4$ & $62.2 \pm 11.6$ & 0.09 \\
\hline Hypertension & $182 / 302(60.3 \%)$ & $392 / 745(52.6 \%)$ & 0.03 \\
\hline Diabetes & $37 / 302(12.3 \%)$ & $116 / 748(15.5 \%)$ & 0.21 \\
\hline Hyperlipidemia & $125 / 302(41.4 \%)$ & $299 / 745(40.1 \%)$ & 0.73 \\
\hline Smoke & $144 / 302(47.7 \%)$ & $325 / 745(43.6 \%)$ & 0.24 \\
\hline Ejection fraction [\%] & $53.2 \pm 9.6$ & $53.6 \pm 11.3$ & 0.70 \\
\hline STEMI & $120 / 303(39.6 \%)$ & $410 / 748(54.8 \%)$ & $<0.001$ \\
\hline Use of GP III & $6 / 85(7.6 \%)$ & $191 / 746(25.6 \%)$ & $<0.001$ \\
\hline Target vessel: & & & 0.32 \\
\hline LM & $1 / 359(0.3 \%)$ & $7 / 699(1.0 \%)$ & \\
\hline LAD & $196 / 359(54.6 \%)$ & $352 / 699(50.4 \%)$ & \\
\hline LCX & $62 / 359(17.3 \%)$ & $136 / 699(19.5 \%)$ & \\
\hline $\mathrm{RCA}$ & $97 / 359(27.0 \%)$ & $190 / 699(27.2 \%)$ & \\
\hline Graft & $3 / 359(0.8 \%)$ & $14 / 699(2.0 \%)$ & \\
\hline Type C lesion & $134 / 320(41.9 \%)$ & $105 / 274(38.3 \%)$ & 0.40 \\
\hline Stent length [mm] & $20.6 \pm 5.2$ & $20.0 \pm 5.4$ & 0.07 \\
\hline Stent diameter [mm] & $3.1 \pm 0.4$ & $3.0 \pm 0.4$ & $<0.001$ \\
\hline
\end{tabular}

BRS - bioresorbable vascular scaffold; EES - everolimus-eluting stent; GP — glycoprotein; LAD — left anterior descending; LCX — left circumflex artery; LM - left main; RCA — right coronary artery; STEMI — ST segment elevation myocardial infarction; P-value: unpaired $t$-test or Fisher's exact test

at 2 years of follow-up, Kaplan-Meier curves demonstrated higher rates of MACE in BRS patients $(9.3 \%$ vs. $4.7 \%, \mathrm{p}<0.001)$. Furthermore, BRS patients were significantly more likely to experience
TLR $(6.1 \%$ vs. $1.9 \%, \mathrm{p}<0.001)$ and TVR $(8.9 \%$ vs. $2.8 \%, \mathrm{p}<0.001)$ than their EES counterparts. Death or MI rates were comparable between the groups $(2.3 \%$ vs. $1.9 \%, \mathrm{p}=0.91 ; 3.7 \%$ vs. $2.8 \%$, 


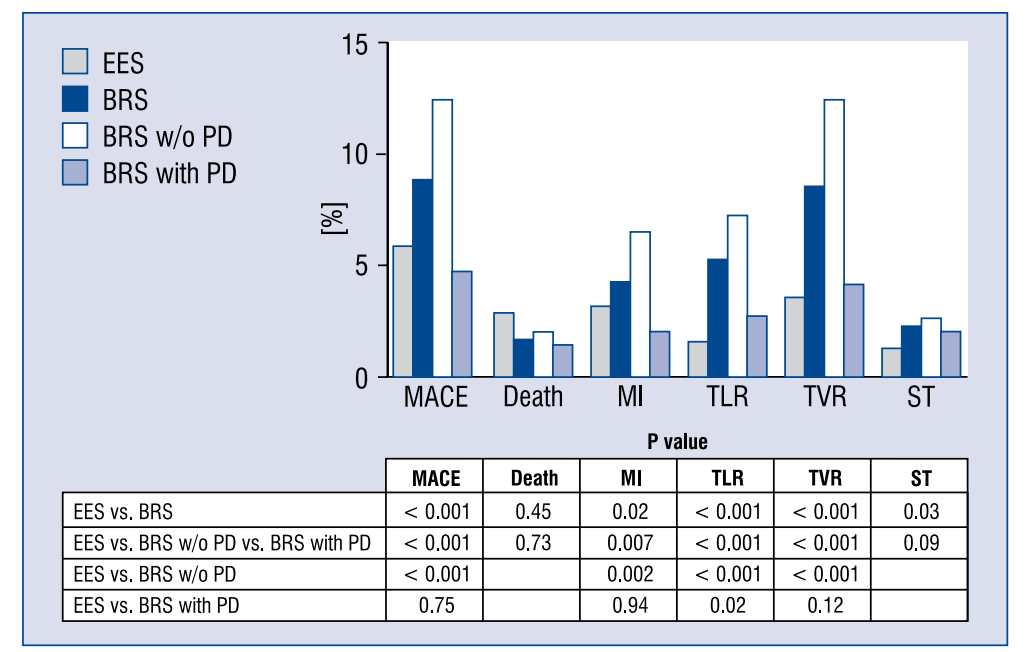

Figure 2. Outcomes at median follow-up for the total study cohort. Comparison between bioresorbable vascular scaffolds (BRS) and everolimus-eluting stents (EES) before and after sensitivity analysis for postdilatation (PD); MACE - major adverse cardiac events (composite of death, myocardial infarction [MI], and target lesion revascularization [TLR]); ST — stent thrombosis; TVR — target vessel revascularization; w/o — without.

Table 2. Baseline characteristics of the matched study cohort (postmatching).

\begin{tabular}{lccc}
\hline & BRS & EES & P \\
\hline Females & $44 / 214(20.6 \%)$ & $42 / 215(19.5 \%)$ & 0.81 \\
Age [years] & $59.7 \pm 13.0$ & $61.5 \pm 11.9$ & 0.18 \\
Hypertension & $120 / 214(56.1 \%)$ & $117 / 215(54.4 \%)$ & 0.77 \\
Diabetes & $30 / 214(14.0 \%)$ & $36 / 215(16.7 \%)$ & 0.50 \\
Hyperlipidemia & $88 / 214(41.1 \%)$ & $92 / 215(42.8 \%)$ & 0.77 \\
Smoke & $110 / 214(51.4 \%)$ & $89 / 215(41.4 \%)$ & 0.04 \\
Ejection fraction [\%] & $51.9 \pm 9.5$ & $53.7 \pm 11.7$ & 0.13 \\
STEMI & $105 / 214(49.1 \%)$ & $97 / 215(45.1 \%)$ & 0.44 \\
Use of GP III & $3 / 46(6.5 \%)$ & $70 / 213(32.9 \%)$ & $<0.001$ \\
Target vessel: & & & 0.001 \\
LM & $1 / 249(0.4 \%)$ & $5 / 212(2.4 \%)$ & \\
LAD & $141 / 249(56.6 \%)$ & $96 / 212(45.3 \%)$ & $38 / 212(17.9 \%)$ \\
$\quad$ LCX & $40 / 249(16.1 \%)$ & $65 / 212(30.7 \%)$ & \\
RCA & $67 / 249(26.9 \%)$ & $8 / 212(3.8 \%)$ & $100 / 240(41.7 \%)$ \\
Graft & $0 / 249(0.0 \%)$ & $19.7 \pm 5.1$ & 0.01 \\
Type C lesion & $111 / 232(47.8 \%)$ & $3.0 \pm 0.4$ & 0.004 \\
Stent length [mm] & $20.8 \pm 5.2$ & $3.1 \pm 0.4$ & \\
Stent diameter [mm] & & \\
\hline
\end{tabular}

BRS - bioresorbable vascular scaffold; EES — everolimus-eluting stent; GP — glycoprotein; LAD — left anterior descending; LCX — left circumflex artery; LM - left main; RCA — right coronary artery; STEMI — ST segment elevation myocardial infarction; P-value: unpaired $t$-test or Fisher's exact test

$\mathrm{p}=0.18$; respectively). ST was also higher in the BRS group (2.8\% vs. $0.9 \%, \mathrm{p}=0.01$ ) (Fig. 3) (Suppl. Table 2 and Suppl. Fig. 2A: see journal website).
In total, $148(49.2 \%)$ of the BRS patients were treated with postdilatation before propensity score matching and 117 (55.2\%) were treated after 


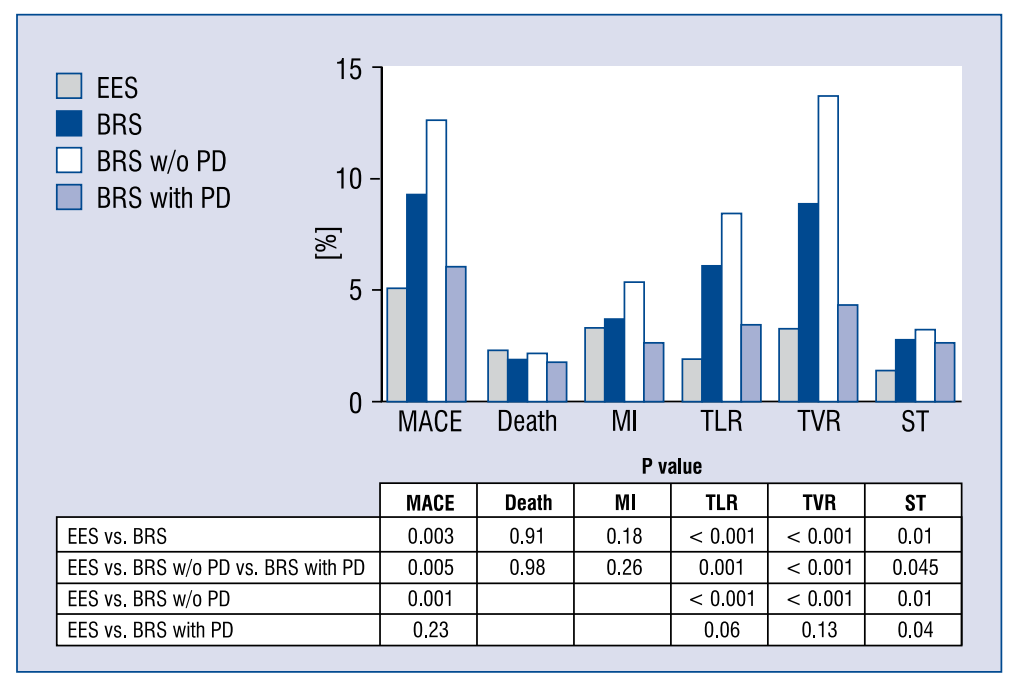

Figure 3. Outcomes at median follow-up for the matched study cohort. Comparison between bioresorbable vascular scaffolds (BRS) and everolimus-eluting stents (EES) before and after sensitivity analysis for postdilatation (PD); MACE - major adverse cardiac events (composite of death, myocardial infarction [MI], and target lesion revascularization [TLR]); ST — stent thrombosis; TVR — target vessel revascularization; w/o — without.

matching (Fig. 1) (Suppl. Table 3 and Suppl. Table 4: see journal website). Of these 148 patients, $95 \%$ were considered as effectively postdilatated using a noncompliant balloon inflated to no more than $0.5 \mathrm{~mm}$ larger than BRS. In the total study cohort, rates of MACE in BRS patients with postdilatation were significantly lower than those without postdilatation and were comparable to those observed in the EES group (4.7\% vs. $12.4 \%$ vs. $5.9 \%$, $\mathrm{p}<0.001$, in BRS with postdilatation, BRS without postdilatation, and EES [the same order throughout]) (Figs. 2, 4) (Suppl. Table 5 and Suppl. Fig. 1B: see journal website).

These differences were mainly driven by lower rates of TLR $(2.7 \%$ vs. $7.2 \%$ vs. $1.6 \%$, $\mathrm{p}<0.001)$. On the other hand, ST rates were only slightly lower in the BRS group with postdilatation but were higher in both the BRS groups than in EES patients $(2.0 \%$ vs. $2.6 \%$ vs. $1.2 \%, \mathrm{p}=0.09)$, although this difference was not significant.

After propensity score matching, KaplanMeier curves confirmed results from the total cohort. The rates of MACE in the BRS patients with postdilatation were again comparable to those observed in the EES group, with both subgroups showing significantly lesser MACE than BRS patients without postdilatation $(6.0 \%$ vs. $12.6 \%$ vs. $4.7 \%, \mathrm{p}<0.001$ ) (Figs. 3, 5) (Suppl. Table 6 and Suppl. Fig. 2B: see journal website).

These differences were also mainly driven by lower rates of TLR (3.4\% vs. $8.4 \%$ vs. $1.9 \%$, $\mathrm{p}<0.001)$. Finally, ST rates were only slightly lower in the BRS group with postdilatation but still remained higher in both the BRS groups than in EES patients (2.6\% vs. $3.2 \%$ vs. $0.9 \%, \mathrm{p}=0.045)$.

\section{Discussion}

The present study provides new insights on actual BRS implantation in ACS patients, demonstrating the crucial role of effective postdilatation. We showed that in long-term follow-up, a) patients with effective postdilatation had similar rates of MACE and TLR compared with EES patients, b) MACE in BRS patients without postdilatation was mainly driven by TLR and was higher than that in EES patients, and c) scaffold thrombosis was not negligible.

In our study, postdilatation was performed using high-pressure noncompliant balloons in $49 \%$ of the patients and these data suggest that optimal postdilatation improves the outcome of BRS implantation, even in the population of ACS with relatively complicated lesions. Postdilatation using a high-pressure balloon is known as the standard deployment technique of contemporary metallic stents because the risk of TVR and ST is related to the final stent dimensions as guided by IVUS [31, 32]. Metallic stents have a stable structure that is able to provide reliable and compliant expansion of the struts without the risk of disruption; however, BRS with polymeric materials has raised concerns 


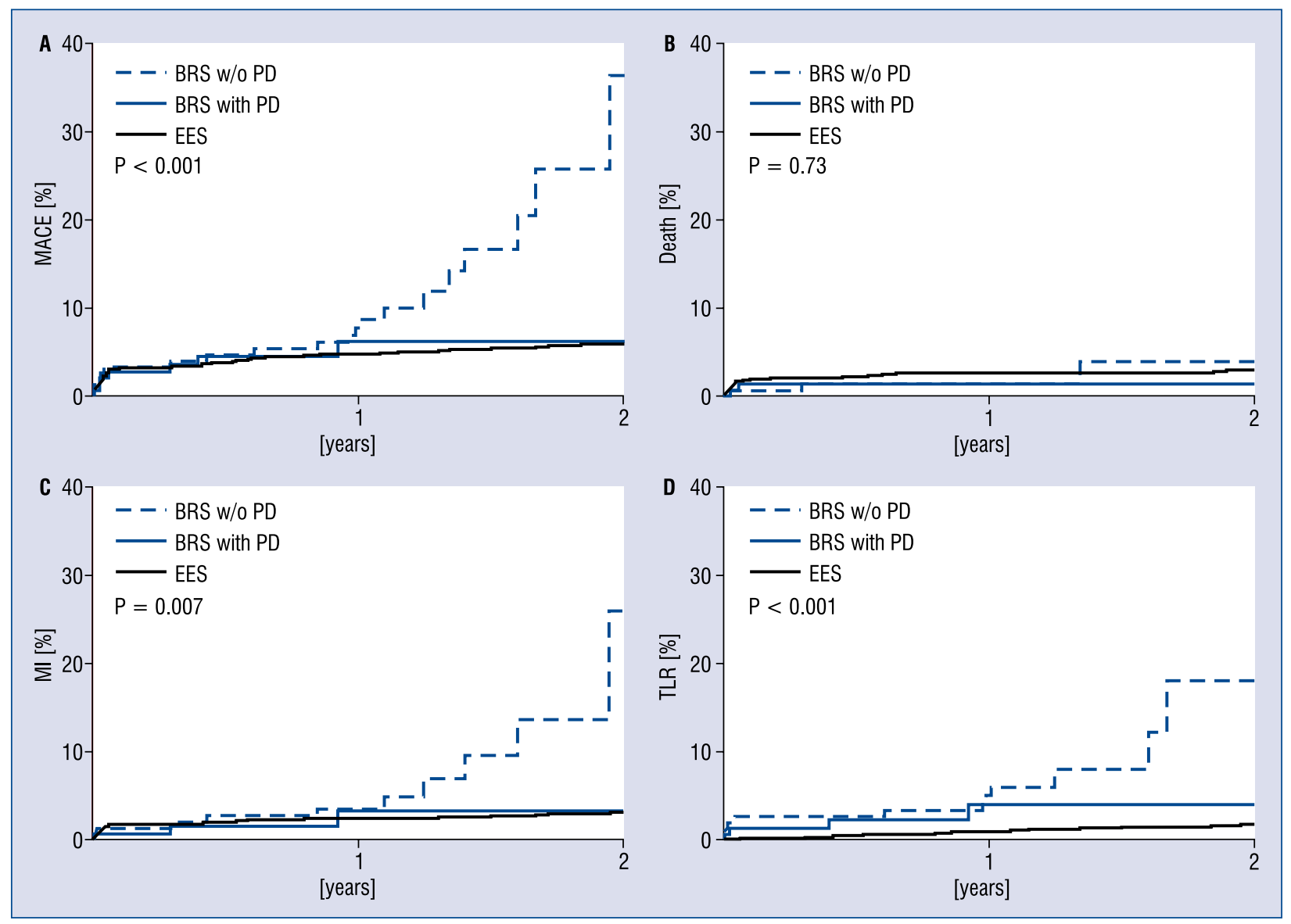

Figure 4. Kaplan-Meier estimates of the outcomes in the total study cohort. Comparison with sensitivity analysis for postdilatation (PD); BRS — bioresorbable vascular scaffold; EES — everolimus-eluting stent; MACE — major adverse cardiac events (composite of death, myocardial infarction [MI], and target lesion revascularization [TLR]); w/o - without; A. MACE; B. Death; C. MI; D. TLR.

regarding over-expansion, disruption, and the effect of postdilatation following its implantation $[27,33]$. For the full expansion of BRS, a recent practical review has recommended that postdilatation should use short noncompliant balloons of no more than $0.5 \mathrm{~mm}$ larger than the nominal scaffold size, although clear and supporting data are not available [25]. The maximum recommended scaffold expansion is no more than $0.5 \mathrm{~mm}$ larger than the nominal scaffold size, which should be followed during the procedure to prevent strut fracture [25]. In the present study, the mean implantation length was longer in BRS patients as compared to EES patients after matching. Type $\mathrm{C}$ lesion was more frequently observed in BRS patients than EES patients, although the difference was not significant. In addition, the use of glycoprotein IIb/IIIa inhibitors was more frequent in EES patients than BRS patients. Indeed, these differences were in favor of EES patients. Moreover,
BRS patients with postdilatation had longer scaffold length, more type $\mathrm{C}$ lesions and less use of glycoprotein IIb/IIIa than BRS patients without postdilatation. Nevertheless, BRS patients with postdilatation had favorable results compared to BRS patients without postdilatation.

Notwithstanding, the rate of scaffold thrombosis was not negligible. In fact, concerns about ST of BRS have recently been raised $[14,15]$. A retrospective study of not only ACS but also stable angina patients showed stent thrombosis can be reduced when guideline-based implantation protocol with appropriate BRS size and pre-and postdilatation was employed [13]. However, in ACS patients of the present study, ST rates still remained higher in both the BRS groups than in EES group. ST occurred more frequently in the subgroup of patients without postdilatation. ST represents an important issue in the ACS setting as it is potentially triggered by enhanced aggregation 


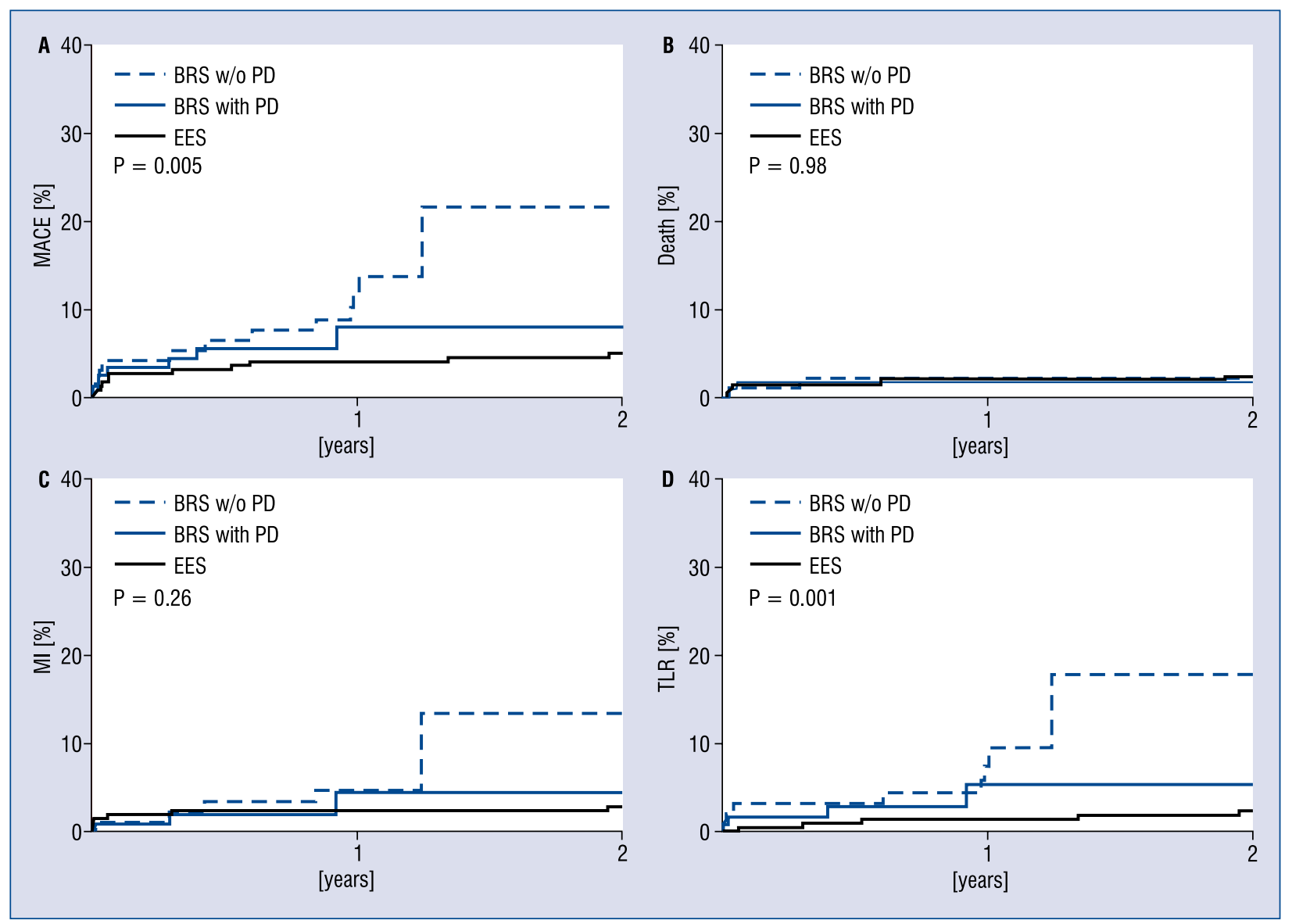

Figure 5. Kaplan-Meier estimates of the outcomes in the matched study cohort. Comparison with sensitivity analysis for postdilatation (PD); BRS — bioresorbable vascular scaffold; EES — everolimus-eluting stent; MACE — major adverse cardiac events (composite of death, myocardial infarction [MI], and target lesion revascularization [TLR]); w/o - without; A. MACE; B. Death; C. MI; D. TLR.

of platelets [34]. BRS represents a potential risk for scaffold thrombosis because of the relatively thick struts and limited expansion. A first randomized trial comparing BRS and EES in STEMI patients with simple lesion showed complete arterial healing with low device-oriented events at 6 months; however, $1.1 \%$ scaffolds thrombosis was reported in early phase [24]. The authors presumed that it was due to an inappropriate matching of the scaffold to the vessel size. Intravascular imaging was used in limited cases in the present study. The imaging techniques especially OCT have higher resolution, so that scaffold integrity, apposition to the underlying wall, presence of thrombus and changes in strut characteristics during the intervention and on follow-up should be studied in the further investigation.

Our results differ from those of previous large randomized trials, possibly because of different patient presentation and different angiographic le- sions. In fact, patients in the trials only presented for unstable angina in $9.8-24 \%$ of the cases and the rates of type $\mathrm{C}$ lesions were $2-20 \%$ (compared with $41.9 \%$ in our complete ACS cohort) [10-12]. The other previous reports on BRS implantation in patients with higher risk lesions showed varying results. On one hand, in the GHOST registry, a large European real-world study of BRS, the prevalence of ACS was $47.4 \%$, including $16.1 \%$ STEMI. Lesion types B2 and C were $23.6 \%$ and $27.6 \%$, respectively. This study showed the annualized rates for cardiac death, target vessel MI, TLR, TVR, and target vessel failure to be $1.6 \%, 3.6 \%$, $7.0 \%, 10.2 \%$, and $11.9 \%$, respectively [19]. On the other hand, some recent reports have shown that BRS may be safely implanted for thrombotic lesions in ACS and STEMI [15-17, 22, 23]. Brugaletta et al. [15] used propensity score matching to show that 1-year results between BRS and EES patients or bare-metal stents (BMS) did not significantly differ. 
In that study, device-oriented endpoints including cardiac death, target vessel MI, and TLR in BRS were similar to those of EES or BMS patients $(4.1 \%$ and $4.1 \%$ [p $=0.99$ ] or $5.9 \%$ [p $=0.31]$, respectively) although scaffolds thrombosis was not negligible. Other recent studies evaluating BRS show different rates of target lesion failure and ST rate. If evaluated closely, these studies show differences in the study population (ACS or not), in the lesion severity and particularly in the postdilatation rates. In fact, the use of postdilatation in BRS studies fluctuated from $14 \%$ in some series to more than $60 \%$ and even up to $90 \%$ in other studies $[10,19,35]$.

\section{Limitations of the study}

The present study has some limitations. First, these data are retrospectively analyzed and derived from nonrandomized evidence, although propensity score matching showed good accuracy (area under the curve of 0.81 [0.76-0.85] and calibration (nonsignificant Hosmer-Lemeshow test). Second, the data concerning dual antiplatelet regimen were not available and the ratio of dual antiplatelet therapy discontinuation was unknown. Third, data at longer follow-up time-points are needed to correctly evaluate performance after the disappearance of the scaffolds.

\section{Conclusions}

Postdilatation appears to be effective when using BRS in patients presenting with ACS and leads to MACE rates that are comparable to those of EES, although scaffold thrombosis is not negligible. Randomized prospective studies are required to further investigate BRS safety and efficacy in ACS.

\section{Conflict of interest: None declared}

\section{References}

1. Moretti C, Quadri G, D'Ascenzo F et al. The STORM (acute coronary Syndrome in paTients end Of life and Risk assesMent) study. Emerg Med J, 2015; 33: 10-16. doi: 10.1136/ emermed-2014-204114.

2. Goto K, Lansky AJ, Ng VG et al. Prognostic value of angiographic lesion complexity in patients with acute coronary syndromes undergoing percutaneous coronary intervention (from the acute catheterization and urgent intervention triage strategy trial). Am J Cardiol, 2014; 114: 1638-1645. doi: 10.1016/j.amjcard.2014.09.003.

3. Brar SS, Leon MB, Stone GW et al. Use of drug-eluting stents in acute myocardial infarction: A systematic review and metaanalysis. J Am Coll Cardiol, 2009; 53: 1677-1689. doi: 10.1016/j. jacc.2009.03.013.
4. Eisenstein EL, Anstrom KJ, Kong DF et al. Clopidogrel use and long-term clinical outcomes after drug-eluting stent implantation. JAMA, 2007; 297: 159-168.

5. Garg S, Serruys PW. Coronary stents: Current status. J Am Coll Cardiol, 2010; 56: S1-S42. doi: 10.1016/j.jacc.2010.06.007.

6. Lotan C, Meredith IT, Mauri L, Liu M, Rothman MT, E-Five Investigators. Safety and effectiveness of the Endeavor zotarolimus-eluting stent in real-world clinical practice: 12-month data from the E-Five registry. JACC Cardiovasc Interv, 2009; 2: 1227-1235. doi: 10.1016/j.jcin.2009.10.001.

7. Krucoff MW, Rutledge DR, Gruberg L et al. A new era of prospective real-world safety evaluation primary report of XIENCE V USA (XIENCE V Everolimus Eluting Coronary Stent System condition-of-approval post-market study). JACC Cardiovasc Interv, 2011; 4: 1298-1309. doi: 10.1016/j.jcin.2011.08.010.

8. Onuma Y, Serruys PW, Perkins LE et al. Intracoronary optical coherence tomography and histology at 1 month and 2, 3, and 4 years after implantation of everolimus-eluting bioresorbable vascular scaffolds in a porcine coronary artery model: An attempt to decipher the human optical coherence tomography images in the ABSORB trial. Circulation, 2010; 122: 2288-2300. doi: 10.1161/CIRCULATIONAHA.109.921528.

9. Serruys PW, Ormiston J, van Geuns RJ et al. A polylactide bioresorbable scaffold eluting everolimus for treatment of coronary stenosis: 5-year follow-up. J Am Coll Cardiol, 2016; 67: 766-776. doi: 10.1016/j.jacc.2015.11.060.

10. Serruys PW, Chevalier B, Dudek D et al. A bioresorbable everolimus-eluting scaffold versus a metallic everolimus-eluting stent for ischaemic heart disease caused by de-novo native coronary artery lesions (ABSORB II): an interim 1-year analysis of clinical and procedural secondary outcomes from a randomised controlled trial. Lancet, 2014; 385: 43-54. doi: 10.1016/S01406736(14)61455-0.

11. Ellis SG, Kereiakes DJ, Metzger DC et al. Everolimus-eluting bioresorbable scaffolds for coronary artery disease. N Engl J Med, 2015; 373: 1905-1915. doi: 10.1056/NEJMoa1509038.

12. Kimura T, Kozuma K, Tanabe $\mathrm{K}$ et al. A randomized trial evaluating everolimus-eluting Absorb bioresorbable scaffolds vs. everolimus-eluting metallic stents in patients with coronary artery disease: ABSORB Japan. Eur Heart J, 2015; 36: 3332-3342. doi: 10.1093/eurheartj/ehv435.

13. Puricel S, Cuculi F, Weissner M et al. Bioresorbable coronary scaffold thrombosis: multicenter comprehensive analysis of clinical presentation, mechanisms, and predictors. J Am Coll Cardiol, 2016; 67: 921-931. doi: 10.1016/j.jacc.2015.12.019.

14. Raber L, Brugaletta S, Yamaji $\mathrm{K}$ et al. Very late scaffold thrombosis: Intracoronary imaging and histopathological and spectroscopic findings. J Am Coll Cardiol, 2015; 66: 1901-1914. doi: 10.1016/j.jacc.2015.08.853.

15. Brugaletta S, Gori T, Low AF et al. Absorb bioresorbable vascular scaffold versus everolimus-eluting metallic stent in ST-segment elevation myocardial infarction: 1-year results of a propensity score matching comparison: The BVS-EXAMINATION Study (bioresorbable vascular scaffold: A clinical evaluation of everolimus eluting coronary stents in the treatment of patients with ST-segment elevation myocardial infarction). JACC Cardiovasc Interv, 2015; 8: 189-1897. doi: 10.1016/j.jcin.2014.10.005.

16. Gori T, Schulz E, Hink U et al. Early outcome after implantation of Absorb bioresorbable drug-eluting scaffolds in patients with acute coronary syndromes. EuroIntervention, 2014; 9: 1036-1041. doi: 10.4244/EIJV9I9A176.

17. Kocka V, Maly M, Tousek P et al. Bioresorbable vascular scaffolds in acute ST-segment elevation myocardial infarction: A prospective multicentre study 'Prague 19'. Eur Heart J, 2014; 35: 787-794. doi: 10.1093/eurheartj/eht545. 
18. Jaguszewski M, Ghadri JR, Zipponi M. Feasibility of second-generation bioresorbable vascular scaffold implantation in complex anatomical and clinical scenarios. Clin Res Cardiol, 2014; 104: 124-135. doi: 10.1007/s00392-014-0757-4.

19. Capodanno D, Gori T, Nef $\mathrm{H}$ et al. Percutaneous coronary intervention with everolimus-eluting bioresorbable vascular scaffolds in routine clinical practice: Early and midterm outcomes from the European multicentre GHOST-EU registry. EuroIntervention, 2014; 10: 1144-1153. doi: 10.4244/EIJY14M07_11.

20. Gori T, Schulz E, Hink U et al. Clinical, angiographic, functional, and imaging outcomes 12 months after implantation of drug-eluting bioresorbable vascular scaffolds in acute coronary syndromes. JACC Cardiovas Interv, 2015; 8: 770-777. doi: 10.1016/j. jcin.2014.12.244.

21. Kraak RP, Hassell ME, Grundeken MJ et al. Initial experience and clinical evaluation of the Absorb bioresorbable vascular scaffold (BVS) in real-world practice: The AMC Single Centre Real World PCI Registry. EuroIntervention, 2015; 10: 1160-1168. doi: 10.4244/EIJY14M08_08.

22. Wiebe J, Mollmann $\bar{H}$, Most A et al. Short-term outcome of patients with ST-segment elevation myocardial infarction (STEMI) treated with an everolimus-eluting bioresorbable vascular scaffold. Clin Res Cardiol, 2014; 103: 141-148. doi: 10.1007/s00392013-0630-x.

23. Diletti R, Karanasos A, Muramatsu T et al. Everolimus-eluting bioresorbable vascular scaffolds for treatment of patients presenting with ST-segment elevation myocardial infarction: BVS STEMI first study. Eur Heart J, 2014; 35: 777-786. doi: 10.1093/ eurheartj/eht546.

24. Sabate M, Windecker S, Iniguez A et al. Everolimus-eluting bioresorbable stent vs. durable polymer everolimus-eluting metallic stent in patients with ST-segment elevation myocardial infarction: Results of the randomized ABSORB ST-segment elevation myocardial infarction-TROFI II trial. Eur Heart J, 2015; 37: 229-240. doi: 10.1093/eurheartj/ehv500.

25. Tamburino C, Latib A, van Geuns RJ et al. Contemporary practice and technical aspects in coronary intervention with bioresorbable scaffolds: A European perspective. EuroIntervention, 2015; 11: 45-52. doi: 10.4244/EIJY15M01_05.

26. Nakatani S, Onuma Y, Ishibashi Y et al. Early (before 6 months), late (6-12 months) and very late (after 12 months) angiographic scaffold restenosis in the ABSORB Cohort B trial. EuroIntervention, 2015; 10: 1288-1298. doi: 10.4244/EIJV10I11A218.
27. De Ribamar Costa J, Jr., Abizaid A, Whitebourn R et al. Impact of post-dilation on the acute and one-year clinical outcomes of a large cohort of patients treated solely with the Absorb Bioresorbable Vascular Scaffold. EuroIntervention, 2015; 11: 141-148. doi: 10.4244/EIJY15M05_06.

28. Hamm CW, Bassand JP, Agewall S et al.; ESC Committee for Practice Guidelines. ESC Guidelines for the management of acute coronary syndromes in patients presenting without persistent ST-segment elevation: The Task Force for the management of acute coronary syndromes (ACS) in patients presenting without persistent ST-segment elevation of the European Society of Cardiology (ESC). Eur Heart J, 2011; 32: 2999-3054. doi: 10.1093/eurheartj/ehr236.

29. Ellis SG, Vandormael MG, Cowley MJ et al. Coronary morphologic and clinical determinants of procedural outcome with angioplasty for multivessel coronary disease. Implications for patient selection. Multivessel Angioplasty Prognosis Study Group. Circulation, 1990; 82: 1193-1202.

30. Applegate RJ, Sacrinty MT, Little WC, Santos RM, Gandhi SK, Kutcher MA. Incidence of coronary stent thrombosis based on academic research consortium definitions. Am J Cardiol, 2008; 102: 683-688. doi: 10.1016/j.amjcard.2008.04.050.

31. Fujii K, Mintz GS, Kobayashi Y et al. Contribution of stent underexpansion to recurrence after sirolimus-eluting stent implantation for in-stent restenosis. Circulation, 2004; 109: 1085-1088. doi: 10.1161/01.CIR.0000121327.67756.19.

32. Colombo A, Hall P, Nakamura S et al. Intracoronary stenting without anticoagulation accomplished with intravascular ultrasound guidance. Circulation, 1995; 91: 1676-1688.

33. Ormiston JA, Serruys PW, Regar E et al. A bioabsorbable everolimus-eluting coronary stent system for patients with single de-novo coronary artery lesions (ABSORB): A prospective open-label trial. Lancet, 2008; 371: 899-907. doi: 10.1016/S01406736(08)60415-8.

34. D'Ascenzo F, Bollati M, Clementi F et al. Incidence and predictors of coronary stent thrombosis: Evidence from an international collaborative meta-analysis including 30 studies, 221,066 patients, and 4276 thromboses. Int J Cardiol, 2013; 167: 575-584. doi: 10.1016/j.jijcard.2012.01.080.

35. Kajiya T, Liang M, Sharma RK et al. Everolimus-eluting bioresorbable vascular scaffold (BVS) implantation in patients with ST-segment elevation myocardial infarction (STEMI). EuroIntervention, 2013; 9: 501-504. doi: 10.4244/EIJV9I4A80. 